\title{
Review on treatment of craniocervical soft tissues arterovenous malformations and hemangiomas
}

\author{
Pierleone Lucatelli ${ }^{1}$, Beatrice Sacconi ${ }^{1}{ }^{, *}$, Michele Anzidei $^{1}$, Mario Bezzi ${ }^{1}$ and Carlo Catalano ${ }^{1}$
}

\begin{abstract}
Vascular malformations include several vascular abnormalities, congenital in most cases, classified according to their dynamic flow characteristics into high-flow and low-flow abnormalities; both types are commonly located in the head and neck region. Imaging modalities such as Echocolor-Doppler, CT, and MRI can be employed in the evaluation of vascular malformations' in order to describe their size, flow velocity, flow direction, and relationship with the surrounding structures, and, even more important, to differentiate between different types of malformations, since treatment modalities differ depending on their nature (low-vs high-flow).
\end{abstract}

Keywords: Vascular malformations, Low-flow vascular malformations, High-flow vascular malformations, Arterovenous malformation, Sclerotherapy

\section{Background}

The term "vascular malformations" includes a large number of vascular abnormalities, originally classified in 1982 by Mulliken \& Glowacki [1]; a modified version proposed in 1992 by Mulliken \& Young is currently the widely used classification to differentiate these malformations in clinical and radiological practice [2] (Fig. 1).

Vascular malformations are congenital in most cases, although not always evident. They usually develop during childhood, increase their dimension following the child growth and show no spontaneous regression. Their growth can be exacerbated during puberty or pregnancy due to hormonal changes, or as a result of thrombosis, infection or trauma. Unlike other vascular abnormalities, they can have an infiltrative behavior, involving multiple tissue planes [3].

Vascular malformations are classified according to their dynamic flow characteristics into high-flow (arteriovenous malformations [AVM] and arteriovenous fistulas [AVFs]) and low-flow abnormalities (venous, lymphatic, capillary, capillary-venous, and capillary-

\footnotetext{
* Correspondence: beatrice.sacconi@fastwebnet.it

${ }^{1}$ Department of Radiological, Oncological and Anatomopathological Sciences

- Radiology, 'Sapienza' University of Rome, Viale Regina Elena 324, 00161 Rome, Italy

${ }^{2}$ Center for Life Nano Science@Sapienza, Istituto Italiano di Tecnologia, Rome, Italy
}

lymphatic-venous); the differential diagnosis between these two groups plays a very important role in the patient management, since malformations with different hemodynamic characteristics follow different treatment pathways [4].

Both high- and low-flow vascular malformation are commonly located in the head and neck region, with the venous and lymphatic malformations ones being located in this region in up to 40 and $80 \%$ of cases respectively, especially in the posterior cervical triangle [5]. AVM and congenital AVF are also frequently localized in the craniocervical region $[6,7]$.

Our purpose is to provide a comprehensive review on management of cranio-cervical vascular malformations, with a special focus on imaging and treatment and their strong interdependency; more in detail, we want to describe the different treatment strategies, and the imaging findings that the radiologists should report before treatment and during the post-procedural follow-up.

\section{Review \\ General features \\ Low-flow vascular malformations}

They are usually classified into venous, lymphatic, capillary and mixed abnormalities. A venous malformation generally consists of small and large dysplastic thin-walled 


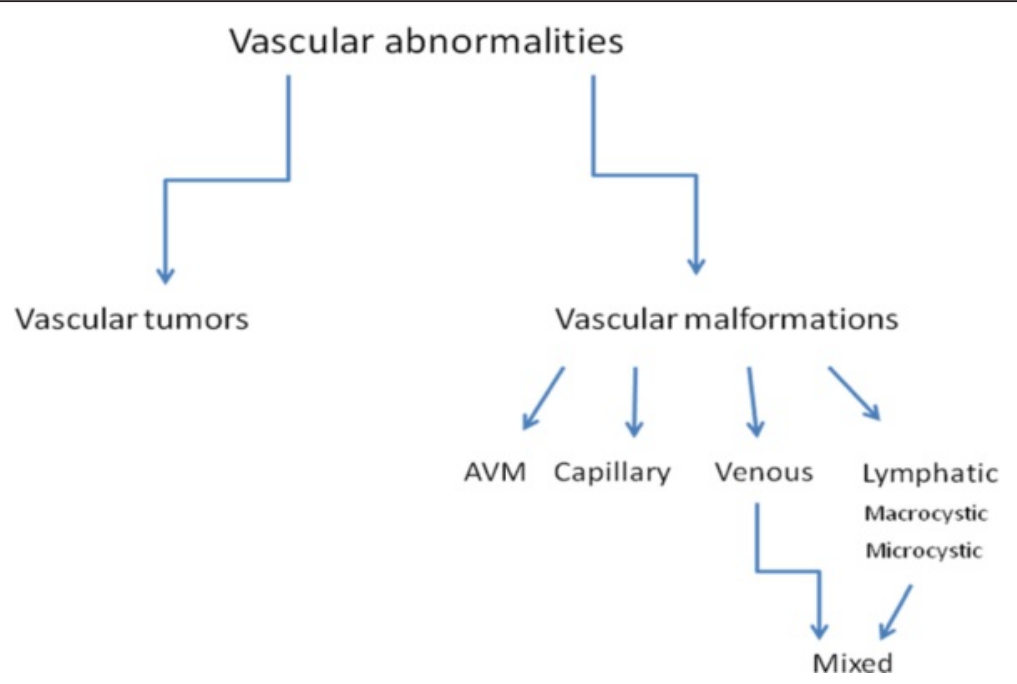

Fig. 1 Modified Mulliken classification for Vascular abnormalities (1992)

venous channels with variable amounts of hamartomatous stroma, thrombi, and phleboliths, and appearing as a blue, soft, non compressible, non pulsatile mass [8]. Lymphatic malformations composed of chyle-filled cysts lined with endothelium and can be divided into microcystic (multiple cysts smaller than $2 \mathrm{~mm}$ ) and macrocystic types (larger cysts) [3]. Capillary malformations are areas of congenital ectasia of thin-walled small vessels of the skin typically confined to the dermis or mucous membranes and appearing as cutaneous red discoloration; they might also be the hallmark of complex anomalies such as Klippel-Trenaunay, Sturge-Weber, and Parkes Weber syndromes [7]. Venous, lymphatic and capillary components can be combined in mixed low-flow malformations.

\section{High-flow vascular malformations}

AVFs are composed by a single vascular channel between an artery and a vein, while AVMs consist of feeding arteries, draining veins, and a nidus formed by multiple dysplastic vascular channels connecting arteries and veins, with absence of a normal capillary bed, usually resulting in an ill-defined mass.

\section{Imaging features}

Multiple imaging modalities should be employed in the evaluation of vascular malformations' characteristics, such as size, flow velocity, flow direction, relationship with the surrounding structures and lesion's appearance and content. US and Echo-Color Doppler usually represent the first imaging techniques to be used, at least in case of superficial vascular lesions, allowing a real-time visualization of arterial and venous flows and flow velocities' measurement. Conventional radiography plays a limited role, being especially useful in evaluating bone (bone erosion or sclerosis, periosteal reaction, and pathologic fracture). Multidetector Computed Tomography (MDCT) permits to evaluate the enhancement pattern of the lesion, thanks to its high temporal resolution, and the presence of thrombosis or calcification. Magnetic Resonance Imaging (MRI) is currently the most valuable modality for the classification of vascular anomalies, allowing to define the extension of vascular lesions and their anatomic relationship to adjacent structures without radiation exposure. Since a functional analysis of the involved vessels is required for treatment planning, the use of Dynamic time-resolved MR angiography has become mandatory (TRICKs, GE or, TWIST, Siemens). These sequences allow the acquisition of images with high temporal and spatial resolution, enabling a clear separation of the arterial inflow from venous drainage and the detection of early venous shunting, and providing information about the contrast material arrival time and the flow direction $[5,9]$.

\section{Low-flow vascular malformations}

In MRI diagnosis of a low-flow malformation is based on the absence of flow voids on SE images and lack of arterial/early venous enhancement on post-contrast sequences; low-flow malformations, especially venous malformations typically show slow gradual filling with contrast material $[7,10]$. In case of hemorrhage or thrombosis, signal heterogeneity can be observed on T1-weighted images, whereas the best sign for identification of a venous malformation is the presence of phleboliths [7]. Delayed contrast-enhanced sequence may demonstrate connections between the malformation and the deep venous system, which can be useful during treatment planning, since it can increase the risk of deep venous thrombosis [3]. Lymphatic malformations 
are usually seen as lobulated, septated masses with high signal intensity on T2-weighted sequences, usually with no post-contrast enhancement in case of microcystic variants and rim and septal enhancement in case of macrocystic lesions. Imaging findings of mixed Malformation may be non-distinguishable from those of venous malformations [3].

\section{High-flow vascular malformations}

MR imaging findings of high-flow malformations include high-flow enlarged feeding arteries and draining veins, appearing as flow voids on SE images or high-signalintensity foci on GRE images, usually as a poorly defined mass. The dynamic enhancement of the AVM is generally well assessed by using time-resolved dynamic 3D MR angiography, with a contrast material rise time of $5-10 \mathrm{~s}$, tipically showing arterial feeders and early venous filling of the lesion $[5,8,10,11]$.

\section{Treatment}

When planning the treatment of any vascular malformations it should be remembered that treatment must be multidisciplinary involving several specialist such as plastic surgeon, vascular surgeon, interventional radiologist and dermatologist. Usually treatment indication is patient's complaint due to the lesion localization (unaesthetic) or for functional (cramps due to stealing syndrome) or locoregional reason (compression of vital structure). Complete eradication of the pathology is rarely achieved after the first procedure; multiple treatment sessions are usually needed,

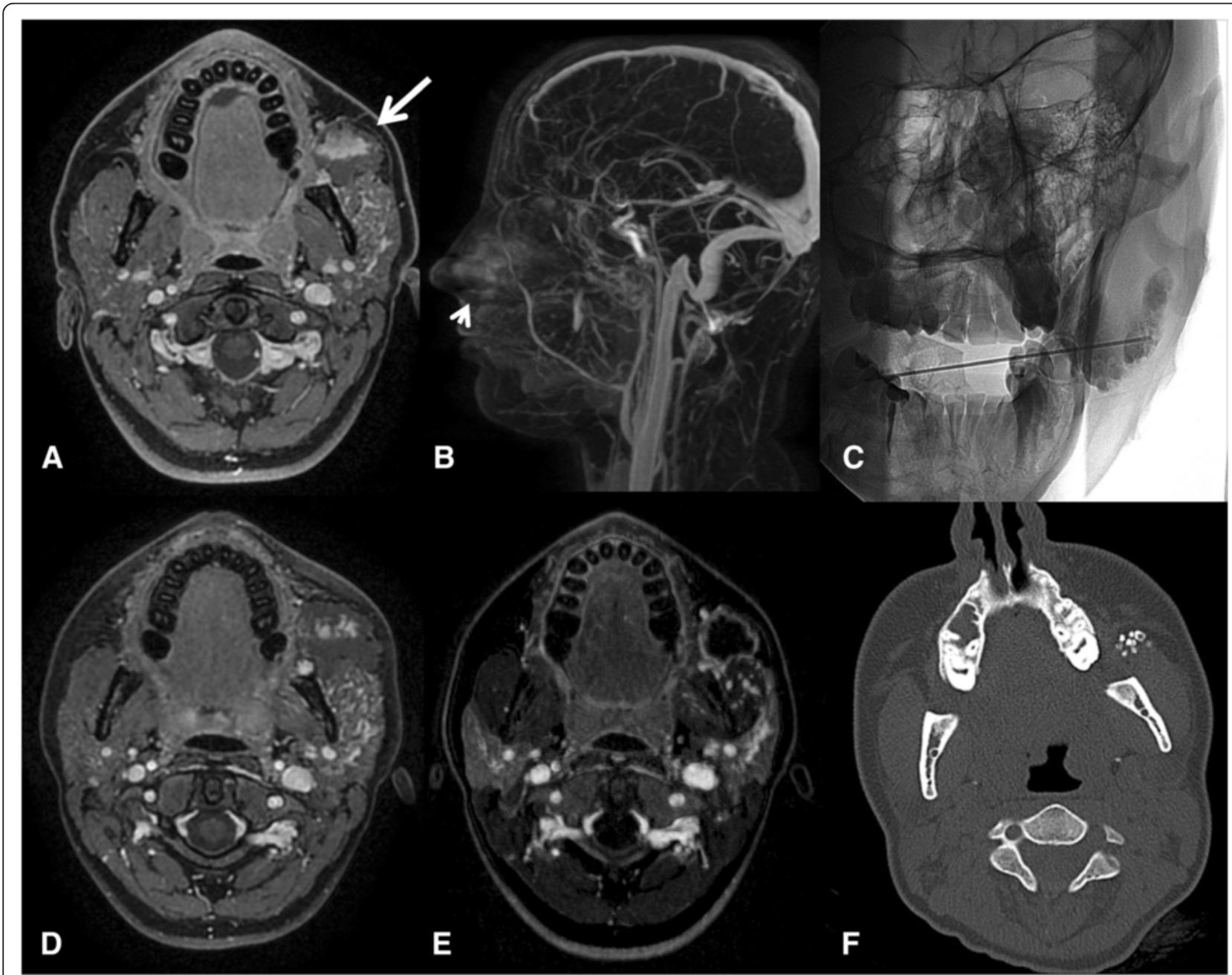

Fig. 2 29-year old female presenting with a venous malformation of the left malar region. a Post-contrast T1-weighted sequence shows partial filling of the lesion with contrast material; (b) TRICKs sequence (MIP) mainly shows the venous drainage into the facial vein; (c) DSA images showing the procedure of percutaneous sclerotherapy; (d) At post-treatment follow up the lesion shows a reduced enhancement on post-contrast T1-weighted images; (e) Follow-up after a new sclerotherapy session: the lesion does not show any central filling, with a peripheral hyperenhancement due to reactive hyperemia (post-contrast T1-weighted images, subtraction tecnique); (f) Late post-treatment follow-up with CT: the lesion has markedly reduced in size and shows internal calcified foci, probably related to residual phleboliths 
even due to the natural tendency of treated vascular malformations to recur. The aim of the treatment is to destroy the nidus of the malformation. Depending on its nature (low- or high-flow) treatment modalities differ $[4,8,9,12]$.

\section{Low-flow malformation}

Low flow malformations are treated percutaneously only if not surgically removable. Due to their usual localization within deep muscles, major surgery is needed to dissected the entire nidus. Percutaneous route is preferred if the nidus is reachable. The nidus is punctured under US guidance or with blind technique (if clinically evident) with a 21 G butterfly needle. Prior to flebography esecution, direct back flow from the nidus should be obtained by gently moving forward or withdrawing the needle tip. Then digital subtraction angiography should be performed in order to observe the anatomy of the nidus and its venous drainage. Careful evaluation of the amount of contrast media needed to opacify the nidus is required in order to identify the correct amount of embolic agent to be injected afterwards. Embolization can be performed only if no direct flow to a drainage vein is seen. Otherwise, needle repositioning is mandatory. The procedure varies depending on the embolic agent employed (atossisclerol vs alchool) after deepening of the analgesia level. Atossisclerol mousse requires less volume in comparison with liquid alcohol, resulting in a moderate pro-thrombotic effect. Alchool provokes immediate thrombosis and edema in the injected vessel by inducing protein denaturation, being more painful than atossisclerol. Antibiotic prophylaxis and corticosteroids in the immediate post-procedural stay are suggested [4, 8, 9, 12] (Fig. 2).

\section{High-flow malformation}

Different approaches are needed to treat an high flow vascular malformation. Treatment options are trans-arterial, trans-venous, or a combination of these with percutaneous embolization. The aim of the treatment is always to obtain exclusion of the nidus. Pre-procedural dynamic MRI helps in choosing the best treatment modality to be used. Arterial approach is usually performed via femoral approach with selective catheterization of the nidus feeder. Microcatethers

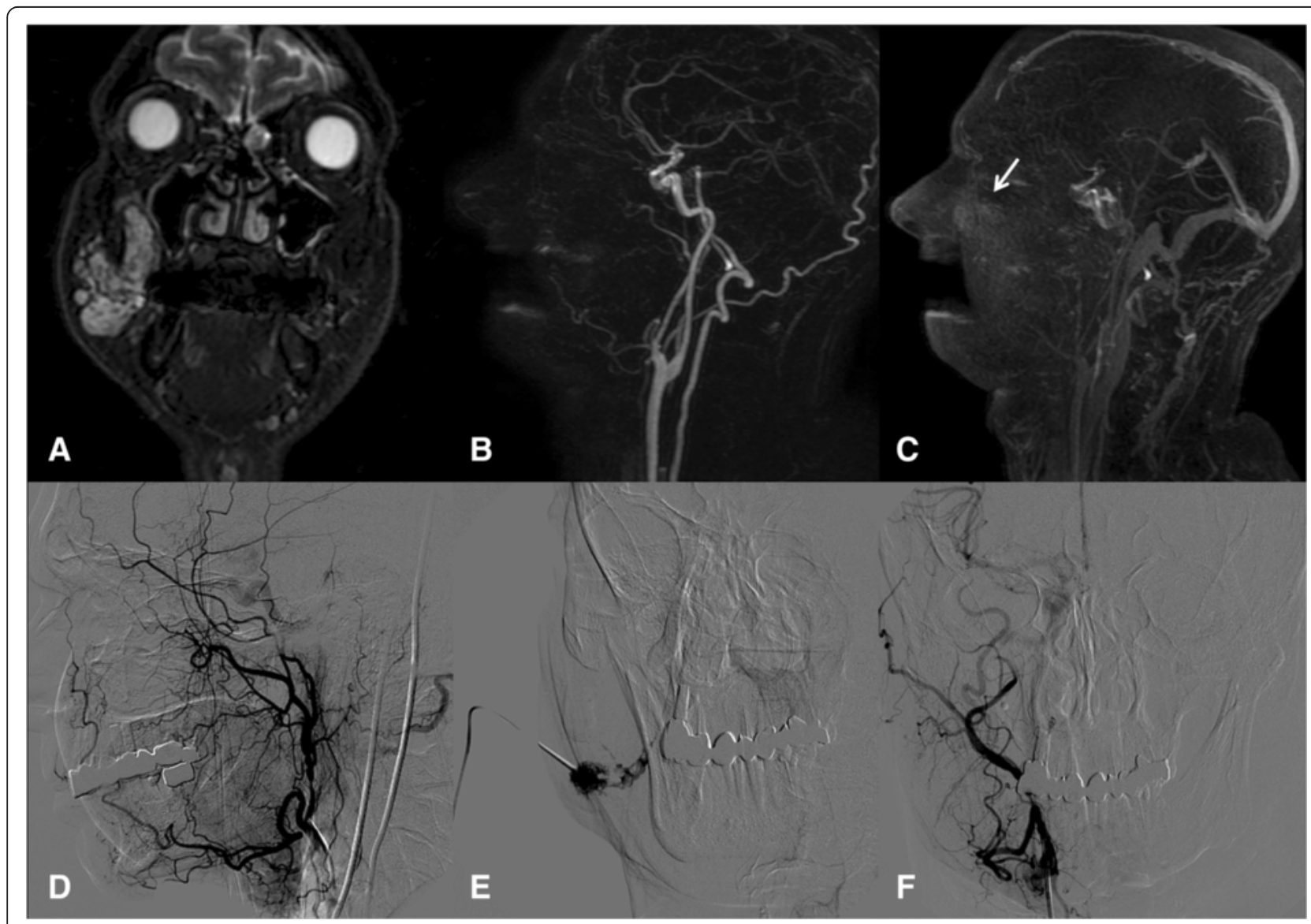

Fig. 338 year-old male, with a vascular malformation of the right malar region; (a) Coronal fat-suppressed T2-weighted sequence showing a large T2-hyperintense lesion of the malar region; (b, c) TRICKS sequence showing the very poor arterial component of the lesion and its gradual filling with contrast material; (d) DSA performed before the sclerotherapy procedure; (e) Percutaneous sclerotherapy (f) The malformation was subsequently treated with chemoembolization 
are required in order to perfom superselective embolization with either glue, foam, particles or coils. Transvenous route is employed when a too fast venous drainage could impair embolic agent deposition within the nidus, thus leading to non target embolization. Occlusion compliant ballons, derived from neuro-intervention procedures, can be used. Percutaneous adjunctive embolization could be needed in case partial opacification or visualization of the nidus are obtained during transvenous/transarterial embolization $[4,8,9,12]$ (Fig. 3).

\section{Post-procedural imaging}

US and MRI are the most useful techniques to assess treatment results and to plan the long-term management strategy $[9,12]$. Imaging technique employed during the follow-up does not differ from the preoperative one in terms of MRI protocol and technical aspects. There is no consensus on timing for the first imaging evaluation after the procedure in literature; at our Institution, post treatment imaging follow-up timeline is usually scheduled within 3 months after the treatment and therefore with longer time interval. However, it is worthy to mention that follow-up timeline could be modified in case of symptoms recurrence.

Treatment-related complications could be minor or major according to the impairment caused to the patient. Minor complications include simple swelling of the treated region, hematoma, partial nidus thrombosis, venous outflow thrombosis. Usually minor complications do not require longer hospitalization time nor adjunctive care. Major complications are usually dependent on treatment modality

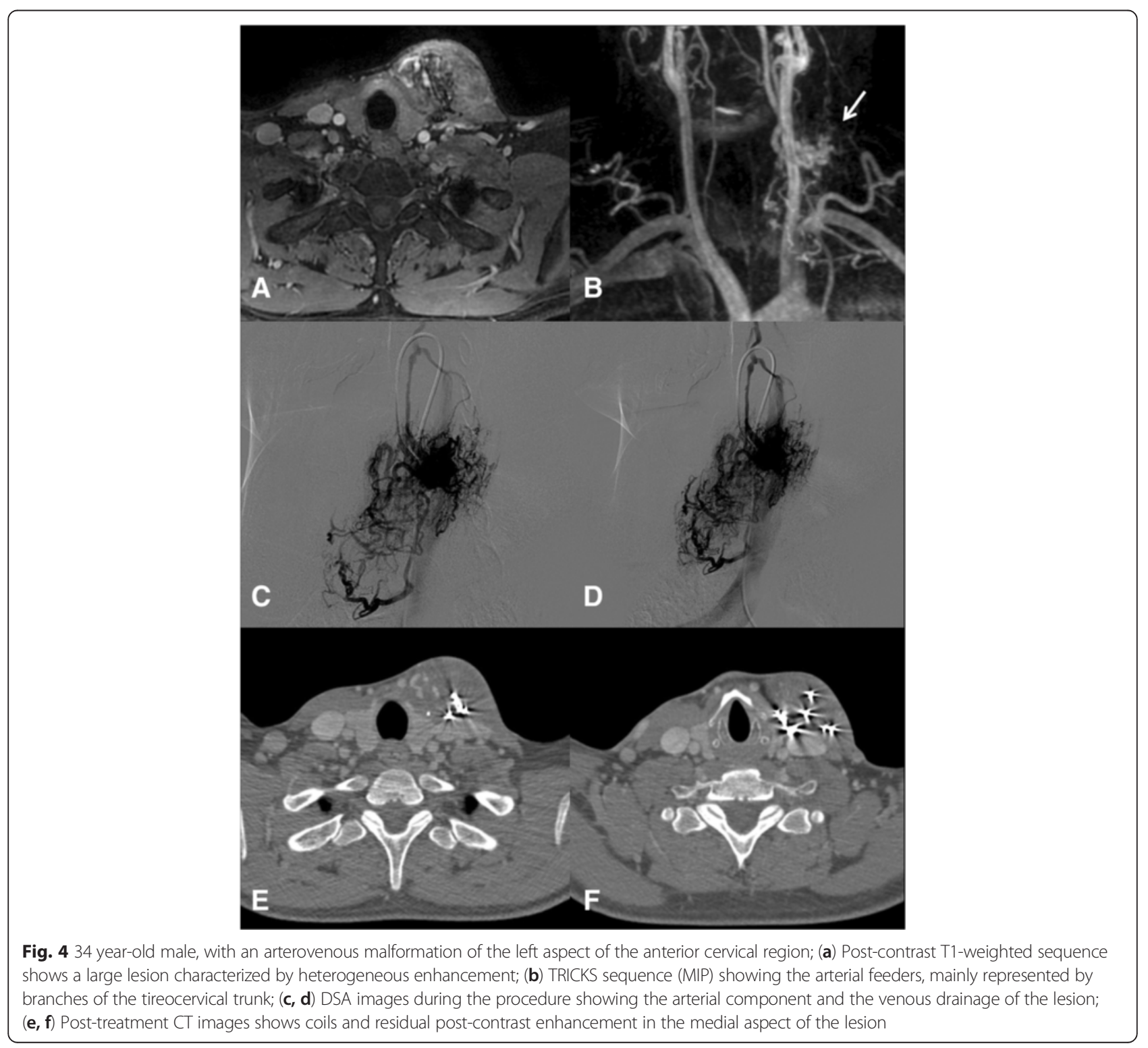


(percutaneous/ endovascular/ combined), on the agent employed (glue, foam, alcohol, coils) and on the district (cranio-facial being the more dangerous). Skin ulceration could be the effect of a not perfect involved injection of embolic agent within the nidus but in the surrounding subcutaneous fat, or to a non target embolization. Nerve paresis is usually due to non target embolization or either compression by swelling of the embolized site and concomitant nerve compression [7-9].

\section{Low-flow malformation}

Ethanol causes almost instantaneous denudation of endothelium with severe inflammatory reaction and thrombosis [7]. Then fibrosis develops and the lesion progressively shrinks. In order to accurately evaluate the therapeutic response after sclerotherapy, the transient inflammatory response needs to be resolved [13]. At MRI, venous malformations after sclerotherapy demonstrate an early high signal intensity related to the inflammatory reaction, associated with no enhancement in the central portion of the treated lesion and peripheral hyperenhancement due to reactive hyperemia [13]. After few months the enhancement usually disappears and a central scar appears as a dark area on both T1-and T2weighted images; a progressive shrinkage of the malformation is frequently observed $[13,14]$.

\section{High-flow malformation}

Since any incomplete treatment may stimulate the lesion's growth and the recruitment of new arterial feeders, the treatment strategy must be planned with the aim of achieving a complete eradication of the nidus [7]. After transarterial embolization, thrombosis of the vascular malformation should be seen; MR angiography may show decreased/absent shunting and reduced/absent venous system's opacification. Any residual component of the malformation must be treated in a second stage. In some cases, Doppler US can be particularly useful during the follow-up, especially in case of ferromagnetic coils have been used; in these cases coils produce artifacts at MRI and MDCT which can hinder an optimal post-procedural evaluation of the malformation [13] (Fig. 4).

\section{Conclusions}

Head and neck region represents one of the most common location for both high- and low-flow vascular malformations, observed in this region in up to 40 and $80 \%$ of cases respectively, especially in the posterior cervical triangle. An accurate pre-procedural depiction of the malformation is mandatory in order to differentiate between high- and low-flow abnormalities and therefore to guide therapeutic decisions; imaging plays an addition role also in the post-procedural follow-up of the treated lesions.

\section{Abbreviations}

AVM: arteriovenous malformations; AVF: arteriovenous fistulas; MRI: magnetic resonance imaging; MDCT: multidetector computed tomography.

\section{Competing interests}

The authors declare that they have no competing interests.

\section{Authors' contributions}

PL, BS: draft editing, literature review, clinical data collection, evaluation of the mri scan (preoperative and post-treatment).MA: draft revision and acceptance, evaluation of the mri scan (preoperative and post-treatment).MB,CC: draft guarantor. All authors read and approved the final manuscript.

Received: 19 October 2015 Accepted: 3 January 2016

Published online: 28 January 2016

\section{References}

1. Mulliken JB, Glowacki J. Hemangiomas and vascular malformations in infants and children: a classification based on endothelial characteristics. Plast Reconstr Surg. 1982;69:412e20.

2. Jackson IT, Carreño R, Potparic Z, Hussain K. Hemangiomas, vascular malformations, and lymphovenous malformations: classification and methods of treatment. Plast Reconstr Surg. 1993;91(7):1216-30.

3. Moukaddam H, Pollak J, Haims AH. MRI characteristics and classification of peripheral vascular malformations and tumors. Skeletal Radiol. 2009;38(6):535-47.

4. McCafferty IJ, Jones RG. Imaging and management of vascular malformations. Clinical Radiology. 2011;66:1208e1218.

5. Dubois J, Alison M. Vascular anomalies: what a radiologist needs to know. Pediatr Radiol. 2010;40(6):895-905.

6. Navarro OM, Laffan EE, Ngan BY. Pediatric soft-tissue tumors and pseudotumors: MR imaging features with pathologic correlation. I. Imaging approach, pseudotumors, vascular lesions, and adipocytic tumors. RadioGraphics. 2009;29(3):887-906.

7. Ernemann U, Kramer U, Miller S, Bisdas S, Rebmann H, Breuninger $H$, et al. Current concepts in the classification, diagnosis and treatment of vascular anomalies. Eur J Radiol. 2010;75(1):2-11.

8. Dubois J, Soulez G, Oliva VL, Berthiaume MJ, Lapierre C, Therasse E. Soft-tissue venous malformations in adult patients: imaging and therapeutic issues. RadioGraphics. 2001;21(6):1519-31.

9. Hyodoh H, Hori M, Akiba H, Tamakawa M, Hyodoh K, Hareyama M. Peripheral vascular malformations: imaging, treatment approaches, and therapeutic issues. RadioGraphics. 2005;25 suppl 1:S159-71.

10. Herborn CU, Goyen M, Lauenstein TC, Debatin JF, Ruehm SG, Kröger K. Comprehensive time-resolved MRI of peripheral vascular malformations. AJR Am J Roentgenol. 2003;181(3):729-35.

11. Anzidei M, Cavallo Marincola B, Napoli A, Saba L, Zaccagna F, Lucatelli $P$, et al. Low-dose contrast-enhanced time-resolved MR angiography at 3T: diagnostic accuracy for treatment planning and follow-up of vascular malformations. Clin Radiol. 2011;66(12):1181-92.

12. Lee BB, Do YS, Yakes W, Kim DI, Mattassi R, Hyon WS. Management of arteriovenous malformations: a multidisciplinary approach. J Vasc Surg. 2004:39(3):590-600.

13. Lucatelli P, Allegritti M, Fanelli F. Chapter 15: Vascular malformations. Catalano C, Anzidei M, Napoli A (eds). Cardiovascular CT and MR Imaging From Technique to Clinical Interpretation. 2013 (ISBN 978-88-470-2868-5).

14. Lucatelli P, Allegritti M, Fanelli F. Chapter 15: Arteriovenous Malformation. Catalano C, Anzidei M, Napoli A (eds). Cardiovascular CT and MR Imaging. 2013 (ISBN 978-88-470-2868-5). 